\title{
Online Transformer Monitoring System
}

\author{
Mohan.S ${ }^{\mathrm{a}, 1}$, S.Vinothkumar ${ }^{\mathrm{b}}$, K.Saravanakumar ${ }^{\mathrm{b}}$, M.Sudarsan ${ }^{\mathrm{b}}$ \\ ${ }^{a}$ Assistant Professor, Department of EEE, \\ ${ }^{b} U G$ Student, Department of EEE, \\ Sri Sairam engineering college, Chennai -600044, Tamil Nadu, India
}

\begin{abstract}
Power transformers, are basically used for stepping up\& down the voltage levels. They are the primary equipment used in power transmission system. So it is primary to maintain all the transformers located geographically, but due to lack of man power it is impossible to monitor regularly. Due to these reasons, if a failure occurs in transformer may cause the network power shutdown. Though there are lot of protection measures that accompany a transformer, but by providing a online monitoring system will increase the reliability and reporting instantaneous fault confidently. This paper gives out the details in design and construction of an automatic monitoring system for power transformer parameters. A node-mcu module [esp8266] was enabled to monitoring of voltage, oil level and temperature (oil \& winding) on a typical power transformer. With the internet of things (IoT), a self-defense system is designed and implemented for the transformer. In this system Transformer parameter are continuously marked and a graph is plotted. If the level of the parameter increases than the actual value, it gives buzzer alarm, if no action taken then the whole system will be tripped safely in power transformers.
\end{abstract}

Keywords. IoT, Transformer, moisture; high temperature.

\section{Introduction}

Power transformer is the single largest costliest equipment with any power sector utility which is indirectly enlightening every place of work and feeding all the means of entertainment in real life [1]. But at the time the most neglected asset, and the future of this valuable assert will be in dark if no appropriate preventive measures taken. Wireless monitoring systems are largely preferred to check various Industrial parameters from different locations. In most of the industries, there is a need to monitor different information for actual creation and all this parameter can't be monitored physically.

Wireless monitoring system is a basic in situations where accessibility to parameter to be monitored in not accessible or the situation where monitoring is a risky one [2]. Now a day's many industries are using wireless monitoring systems, but in our project which concentrate on power transformers. Power transformers are outfitted with some least amount protection that ensures their protection and dependable operation. It is very important that power transformer 's breakdown is minimized to the extreme because of their frequent maintenance, but many transformer are not maintained due to

\footnotetext{
${ }^{1}$ Mohan.S, Assistant Professor, Department of EEE, Sri Sairam engineering college, Chennai -600044, Tamilnadu, India; E-mail: mohan.eee@sairam.edu.in.
} 
various reasons like they are present in city outer, remote area etc, by installing wireless monitoring system we can monitor any types of transformer. Wireless monitoring systems are largely preferred to check various Industrial parameters from different locations [3]. In most of the industries, there is a need to monitor different information for actual creation and all this parameter can't be monitored physically. But at the time the most neglected asset, and the future of this valuable assert will be in dark if no appropriate preventive measures taken. Wireless monitoring systems are largely preferred to check various Industrial parameters from different locations. In most of the industries, there is a need to monitor different information for actual creation and all this parameter can't be monitored physically.

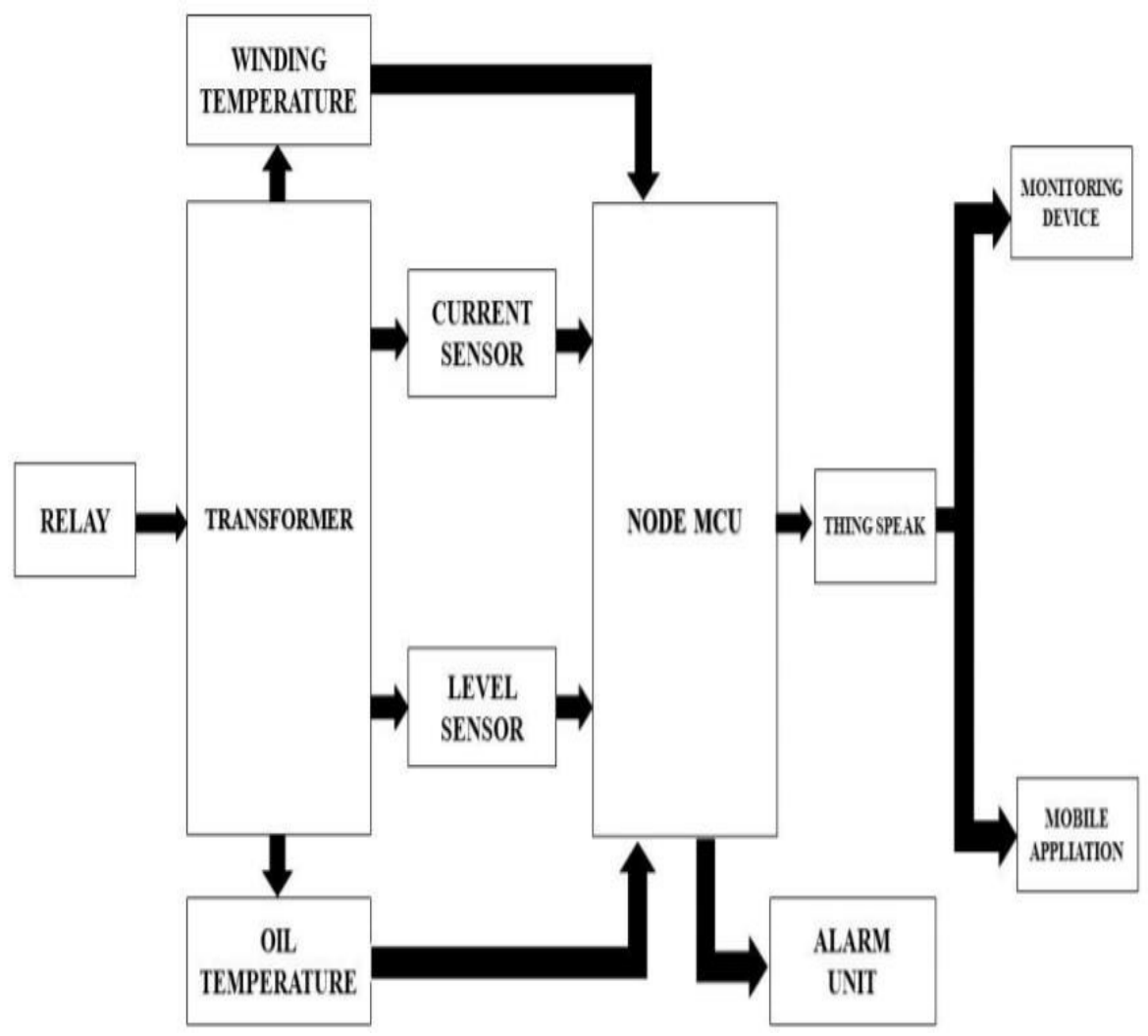

Figure 1. Block diagram

\section{Working}

The main objective of our project online transformer monitoring systems is to monitor the oil level, winding temperature, oil temperature and output current [4]. It also safeguards the power transformer in Figure 1. Firstly, Thermostat, current sensor and ultrasonic sensor are incorporated to various parts of the transformer [5]. Then, things view free application is installed to the system for monitoring purpose. Later the user will Have to login to the application using their login credentials Then the 


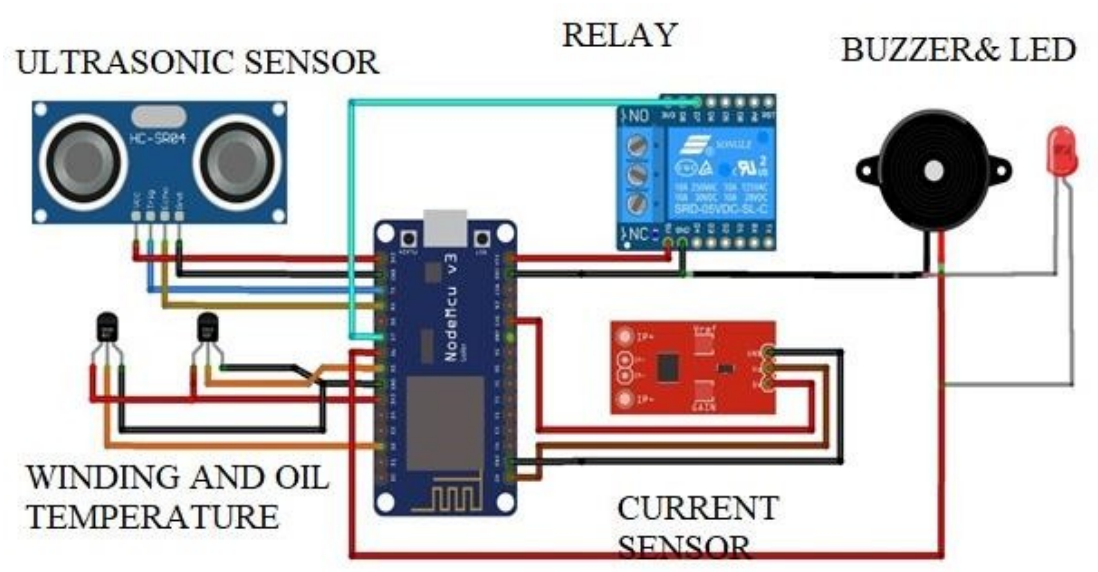

NODE MCU

Figure 2. Circuit diagram

application will be synchronized to the sensors by the means of node-mcu. In Figure 2, the ultrasonic sensor, placed in the conservative tank of the power transformer monitors the oil level. Predetermined value of oil level is fed to the application priory.

As the sensor senses the oil level, it is simultaneously plotted into graph by the application. If the level drops or exceeds the predetermined value, the alert is thrown to the user and the alarm rings, if the alert is not noticed by the user for a period of time the particular unit will be disconnected by relay which is connected to the primary side of the transformer. A current sensor is connected in series to the load to monitor the output current

The winding temperature is monitored by thermostat which is placed with the analog meter. And similarly another thermostat is placed to analog meter corresponding to the oil temperature, the respective graphs are plotted in the application and the user is given an alert as mentioned earlier. If the temperature (oil and winding) further increases the primary side of the transformer is disconnected safely.

\section{Cloud}

All the data have been send to the cloud using MQTT protocol. It uses mosquito protocol. Any user can be able to access it using user id and password. According to cloud data all the actions could be taken. Cloud helps us to know about real time data analysis of sensor value and here uses the thing-speak online server which is interlinked with the Mat works. In this we have given a delay loop hole of 2 seconds for every duty cycle the system refreshes and the resultant of them is to be monitored on the graphical view in the MQTT protocol, which make sense of the data in the periodical data and the time wise. 


\section{Alternating System}

For the sake of the transformer health the system is given a limitation of the temperature of the oil and winding which senses and in case of the winding which senses and in case of the oil and winding which senses and in case of the outbreak of the given level it intimates the user by giving a emergency alert in Figure 3 to Figure 6.

\section{Results}

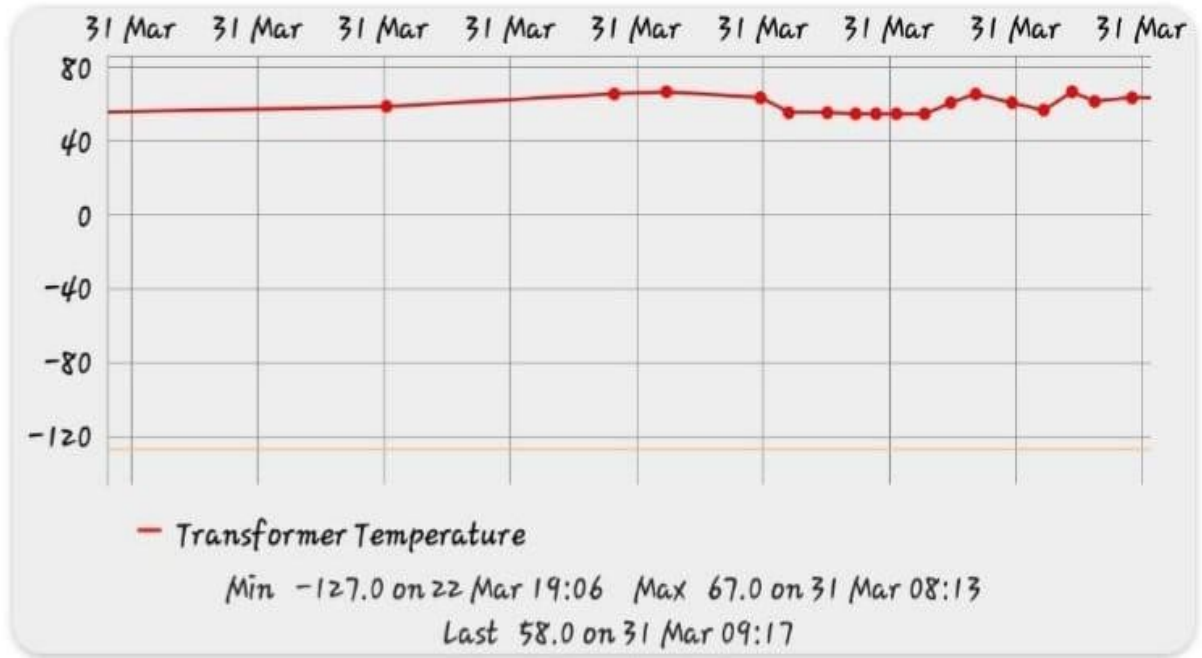

Figure 3. Transformer temperature

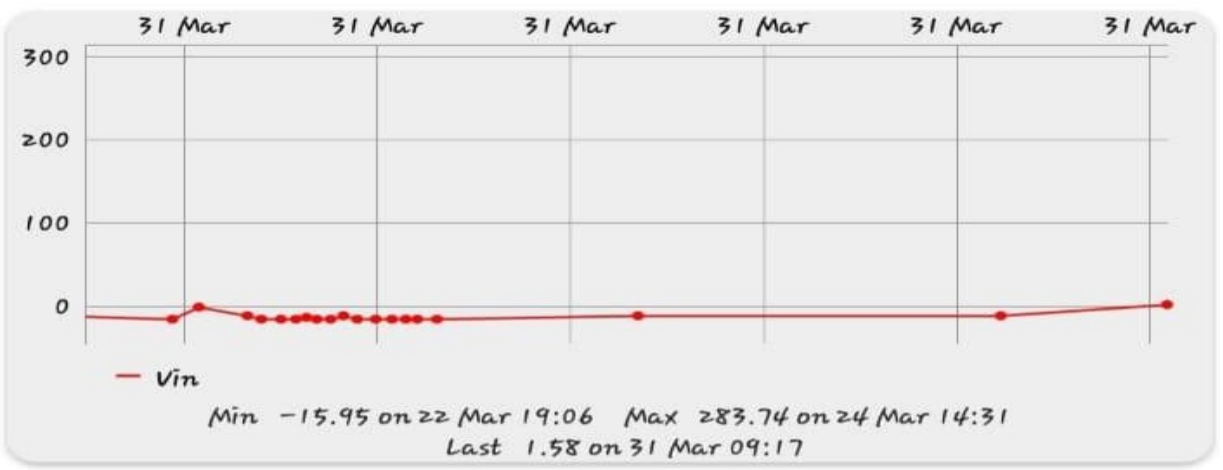

Figure 4. Voltage measured 


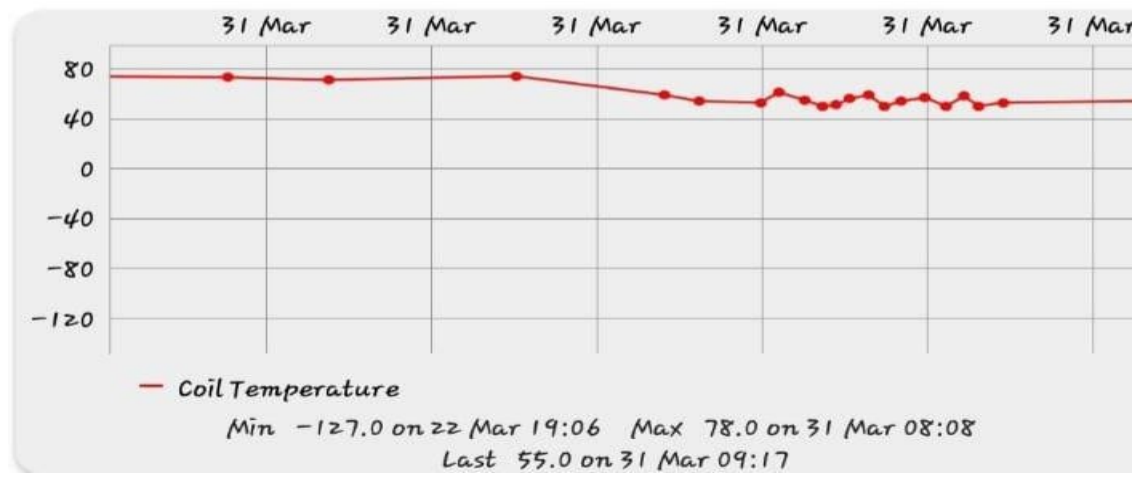

Figure 5. Finding temperature

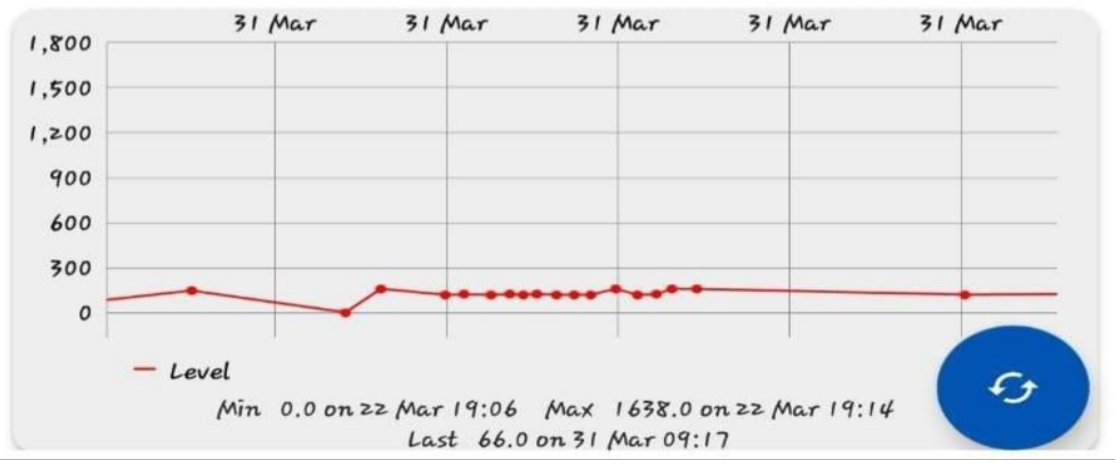

Figure 6. Oil level

\section{Hardware Implementation}

The hardware model is implemented for prototype of transformer monitoring the results are obtained through mobile application and system output in Figure 7. The proto type model implemented is shown below

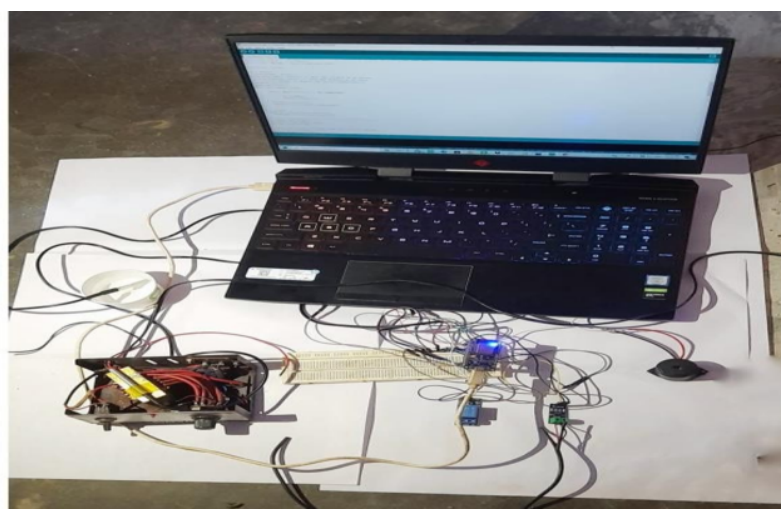

Figure 7. Hardware implementation 


\section{Conclusion}

This paper has projected system arrangement which is commercial and replaced the errors that would occur by manual transformer monitoring set-up. This system provides a cloud-based storage and is accessible through a web application where the data is reachable distantly. There is image and hearing alert method to inform sub-station condition. In addition to the arrangement provides a path way to carry out necessary measure in case of urgent situation for the transformers. The projected arrangement saves cost very much and improving dependability.

\section{References}

[1] Santhosh, C., Aswin Kumer, S. V., Gopi Krishna, J., Vaishnavi, M., Sairam, P., \& Kasulu, P. (2021). IoT based smart energy meter using GSM. Materials Today: Proceedings. https://doi.org/10.1016/j.matpr.2021.02.641

[2] Kaur, M., Mathew, L., Alokdeep, \& Kumar, A. (2018). Implementation of Smart Metering based on Internet of Things. IOP Conference Series: Materials Science and Engineering, 331, 012015. https://doi.org/10.1088/1757-899X/331/1/012015

[3] Barman, B. K., Yadav, S. N., Kumar, S., \& Gope, S. (2018). IOT Based Smart Energy Meter for Efficient Energy Utilization in Smart Grid. 2018 2nd International Conference on Power, Energy and Environment: Towards Smart Technology (ICEPE), 1-5. https://doi.org/10.1109/ EPETSG.2018.8658501

[4] Avancini, D. B., Rodrigues, J. J. P. C., Rabêlo, R. A. L., Das, A. K., Kozlov, S., \& Solic, P. (2021). A new IoT-based smart energy meter for smart grids. International Journal of Energy Research, 45(1), 189-202. https://doi.org/10.1002/er.5177

[5] Hlaing, W., Thepphaeng, S., Nontaboot, V., Tangsunantham, N., Sangsuwan, T., \& Pira, C. (2017). Implementation of WiFi-based single phase smart meter for Internet of Things (IoT). 2017 International Electrical Engineering Congress (IEECON), 1-4. https://doi.org/10.1109/IEECON.2017.8075793 